\title{
Evaluation of Erythromycin as a Tool to Assess CYP3A Contribution of Low Clearance Compounds in a Long-Term Hepatocyte Culture
}

\author{
Tom S. Chan, Young-Sun Scaringella, Klairynne Raymond, and Mitchell E. Taub \\ Boehringer Ingelheim Pharmaceuticals Inc., Drug Metabolism and Pharmacokinetics, Ridgefield, Connecticut
}

Received February 20, 2020; accepted April 29, 2020

\begin{abstract}
Long-term hepatocyte culture systems such as HepatoPac are well suited to evaluate the metabolic turnover of low clearance (CL) drugs because of their sustained metabolic capacity and longerterm viability. Erythromycin (ERY), a moderate, mechanism-based inhibitor of CYP3A, was evaluated as a tool in the HepatoPac model to assess contribution of CYP3A to the clearance of drug candidates. ERY inhibited CYP3A activity by $58 \%$ and $80 \%$ at 3 and $10 \mu \mathrm{M}$, respectively, for up to 72 hours. At $30 \mu \mathrm{M}$, ERY inhibited midazolam hydroxylation by $>85 \%$ for the entire 144-hour duration of the incubation. Alprazolam $\mathrm{CL}_{\text {int }}$ was inhibited $58 \%$ by $3 \mu \mathrm{M}$ of ERY, $75 \%$ by $15 \mu \mathrm{M}$ of ERY, $89 \%$ by $30 \mu \mathrm{M}$ of ERY, and $94 \%$ by $60 \mu \mathrm{M}$ of ERY. ERY $(30 \mu \mathrm{M})$ did not markedly affect $\mathrm{CL}_{\text {int }}$ of substrates for several other major cytochrome $\mathbf{P 4 5 0}$ isoforms evaluated and did not markedly inhibit uridine diphosphoglucuronosyl transferase (UGT) isoforms 1A1, 1A3, 1A4, 1A6, 1A9, 2B7, or 2B15 as assessed using recombinant UGTs. ERY only mildly increased CYP3A4 gene
\end{abstract}

Introduction

Cytochrome P450s (P450) are drug-metabolizing enzymes that contribute to the elimination of approximately $45 \%$ of marketed drugs (Jones et al., 2009; Cerny, 2016). CYP3A continues to play a dominant role as the $\mathrm{P} 450$ isoform most often involved in the metabolism of drugs (Guengerich, 1999; Zhang et al., 2007; Zientek and Youdim, 2015).

Determining the contribution of $\mathrm{P} 450$ isoform metabolism to the total body clearance of drug candidates is required to predict the susceptibility of a drug to changes in exposure caused by inhibition or induction of its metabolism by comedicants (drug-drug interactions, DDI). Typical in vitro reaction phenotyping methodologies include monitoring the depletion of parent compound, or monitoring metabolite formation, in recombinantly expressed $\mathrm{P} 450$ enzymes and/or human-derived microsomes in the presence and absence of isoform-selective inhibitor (Yan and Caldwell, 2013). The successful use of these methods to reliably predict clinical DDIs is contingent on a number of important factors, such as having sufficient metabolism of the drug to observe parent depletion or metabolite formation, and the selectivity of P450 inhibitors used. In addition, data generated from experiments in which recombinantly expressed enzymes are employed must then be considered in the context of appropriate scaling factors to reflect the relative abundance of

https://doi.org/10.1124/dmd.120.090951 expression by 2.1 -fold (14\% of rifampicin induction) at $120 \mu \mathrm{M}$, indicating that at effective concentrations for inhibition of CYP3A activity (30-60 $\mu \mathrm{M})$, arylhydrocarbon receptor, constitutive androstane receptor, and pregnane-X-receptor activation are not likely to markedly increase levels of other drug-metabolizing enzymes or transporters. ERY at concentrations up to $60 \mu \mathrm{M}$ was not toxic for up to 6 days of incubation. Use of ERY to selectively inhibit CYP3A in high-functioning, long-term hepatocyte models such as HepatoPac can be a valuable strategy to evaluate the contribution of CYP3A metabolism to the overall clearance of slowly metabolized drug candidates.

\section{SIGNIFICANCE STATEMENT}

This work describes the use of erythromycin as a selective inhibitor of CYP3A to assess the contribution of CYP3A in the metabolism of compounds using long-term hepatocyte cultures.

ABBREVIATIONS: AhR, arylhydrocarbon receptor; ALT, alanine transaminase; APZ, alprazolam; CAR, constitutive androstane receptor; CL, Clearance; $\mathrm{CL}_{\text {int }}$, intrinsic clearance; DDI, drug-drug interaction; DMXAA, 5,6-dimethyxanthenone-4-acetic acid; ERY, erythromycin; HLM, human liver microsome; KTZ, ketoconazole; MDZ, midazolam; MRM, multiple reaction monitoring; P450, cytochrome P450; PCR, polymerase chain reaction; P-gp, P-glycoprotein (MDR-1); PXR, pregnane-X-receptor; RIF, rifampicin; UGT, uridine diphosphoglucuronosyl transferase. 


\section{Materials and Methods}

HepatoPac cultures (Lots 3121B, 4202, 4297, 1307, ACR) were purchased from BioIVT Inc. Donor demographics are shown in Table 1. APZ, 6-(4Chlorophenyl)imidazo[2,1-b][1,3]thiazole-5-carbaldehyde O-(3,4-dichlorobenzyl)oxime (CITCO), diazepam, omeprazole (OME), rifampicin (RIF), tolbutamide, riluzole, risperidone, and erythromycin were purchased from Sigma Aldrich. Nevirapine was obtained from Boehringer Ingelheim Pharmaceuticals.

Cell Culture. HepatoPac plates were received from BioIVT in sealed 24or 96-well polystyrene plates in warm packaging. A cell count value of 3350 hepatocytes/well was used to calculate $\mathrm{Cl}_{\text {int }}$ in a 96-well plate format. The shipment media was replaced with $64 \mu 1 /$ well of warmed HepatoPac maintenance media containing $10 \%$ FBS for a 96 -well plate format or $400 \mu \mathrm{l} /$ well for a 24 -well plate format. Incubation conditions were $37^{\circ} \mathrm{C}, 90 \%$ humidity, and $10 \% \mathrm{CO}_{2}$. After 2 to 3 days, the wells were washed with serum-free HepatoPac media and then filled with $32 \mu \mathrm{l}$ of serum-free HepatoPac media (for earlier studies using donors $1307,3121 \mathrm{~B}$, and 4202) or Hepatopac media containing 5\% serum (for later studies using 4297 and ACR). After a 15-minute preincubation, $32 \mu \mathrm{l}$ of compound at $2 \times$ concentration was added to each well. Final incubation concentrations were as follows: APZ and diazepam were $0.1 \mu \mathrm{M}$, risperidone and tolbutamide were $0.2 \mu \mathrm{M}$, riluzole was $0.4 \mu \mathrm{M}$, KTZ was 1 and $3 \mu \mathrm{M}$, and ERY was 3,10, 15, 30, or $60 \mu \mathrm{M}$.

The rate of midazolam 1'hydroxylation was also measured after 12, 24, 48, 72, 100, and 144 hours after ERY treatment $(3,10$, and $30 \mu \mathrm{M})$. This study was carried out in duplicate on a 96-well culture plate using donor 1307. After each time point, media containing ERY was removed and replaced with serum-free media containing midazolam $(15 \mu \mathrm{M})$. After a period of 2,4 , and 6 minutes after midazolam addition (two wells/time point), the reaction was stopped by the addition of stop solution as described below.

In all incubations, the concentration of organic solvent (methanol or acetonitrile) did not exceed $0.52 \%(\mathrm{v} / \mathrm{v})$.

At selected time points based on prior studies (Chan et al., 2013), $192 \mu$ l of stop solution containing $0.1 \mu \mathrm{M}$ nevirapine in $60 \%$ acetonitrile, $59.9 \%$ water, and $0.1 \%$ acetic acid was added to a set of wells. The contents of each of the wells was triturated and transferred to a 96-well, 0.1- $\mu \mathrm{m}$ glass fiber filter plate fitted on top of a 96-deep well collection plate. Samples were centrifuged at $3000 \mathrm{~g}$ for 15 minutes. The filtrates were injected into the mass spectrometer for quantitation.

$\mathrm{CL}_{\text {int }}$ was calculated based on the following equation:

$$
C L_{\text {int }}=k_{\text {el }} \frac{64 \mu \mathrm{l}}{3350 \mathrm{cells}} \times 1,000,000 \mathrm{cells}
$$

where $k_{e l}$ is the elimination rate constant. Percent inhibition was calculated based on the following equation.

$$
\% \text { inhibition }=100 \times\left[1-\left(\frac{C L_{\text {int, }, \text { inhibited }}}{C L_{\text {int }, \text { control }}}\right)\right]
$$

where $\mathrm{CL}_{\text {int, inhibited }}$ is the $\mathrm{CL}_{\text {int }}$ of the compound in the presence of the CYP3A inhibitor and $\mathrm{CL}_{\mathrm{int}}$, control is the $\mathrm{CL}_{\mathrm{int}}$ of the compound in the absence of the inhibitor.

Viability. HepatoPac cultures (lot ACR) were incubated with ERY at 0, 10, 30, and $60 \mu \mathrm{M}$ or acetaminophen at $20 \mathrm{mM}$ for 144 hours. Viability was assessed periodically by morphologic assessment using an Evos inverted microscope with a $20 \times$ phase contrast objective (Thermo Fisher Scientific). Cell layer samples were also collected at 2, 4, 24, 48, 96, and 144 hours from each well, and hepatocellular alanine transaminase (ALT) was measured indirectly by using an ALT spectrophotometric assay kit (Biovision Inc.) that measures the activity of ALT via conversion of alanine to pyruvate.

Nuclear Receptor Activation. Arylhydrocarbon receptor (AhR), constitutive androstane receptor (CAR), and pregnane-X-receptor (PXR) activation, indirectly followed by monitoring CYP1A2, CYP2B6, and CYP3A4 induction, was assessed in the presence of $10,30,60$, and $120 \mu \mathrm{M}$ of ERY or positive controls OME (50 $\mu \mathrm{M}$; CYP1A2), CITCO (0.1 $\mu \mathrm{M}$; CYP2B6), and RIF (10 $\mu \mathrm{M}$; CYP3A4). HepatoPac cultures consisting of a pool of 10 donors (Lot ACR) were used in a 24-well plate format. ERY or positive controls were incubated for 48 hours. Media with inducer was refreshed after 24 hours of incubation. The solvent used was acetonitrile, which did not exceed $0.5 \%$ of the incubation media. Cells were harvested in lysis buffer and mRNA was purified using the mRNA
Catcher Plus system (Invitrogen). Reverse transcription was carried out using Superscript VILO mastermix (Invitrogen) on a Gene Amp 9700 thermocycler (Applied Biosystems). CYP1A2, 2B6, and 3A4 gene expression was assessed with real-time polymerase chain reaction, using a QuantStudio Flex 7 system (Applied Biosystems). Amplification was carried out by using commercially available Taqman assays for CYP1A2 (Hs00167927_m1), 2B6 (Hs00167937_g1), and 3A4 (Hs00604506_m1) and human glyceraldehyde3-phosphate dehydrogenase (Hs02786624_g1) as the housekeeping gene. Gene expression was determined by using the $\Delta \Delta$ cycle-threshold method (Livak and Schmittgen, 2001).

Effect of ERY on the Activity of Recombinant UGTs. Recombinant UGTs (Supersomes; Corning Inc.) were incubated in $0.1 \mathrm{M}$ Tris buffer, $\mathrm{pH} 7.8$, with $5 \mathrm{mM} \mathrm{MgCl}_{2}$ and $25 \mu \mathrm{g} / \mathrm{mg}$ alamethecin. The protein concentrations used ranged from 0.083 to $1 \mathrm{mg} / \mathrm{ml}$ depending on the recombinant UGT. For UGTs $1 \mathrm{~A} 1$ and 1A3, activity was assessed using $150 \mu \mathrm{M}$ of b-estradiol. For UGT1A4, activity was assessed using $200 \mu \mathrm{M}$ of trifluoperazine. For UGTs 1A6, 1A9, 2B7, and 2B15, activity was assessed using 7-hydroxy-4-(trifluoromethyl) coumarin. Incubations containing UGT substrates in the presence or absence of 30 or $60 \mu \mathrm{M}$ of ERY were initiated by the addition of uridine diphosphoglucuronic acid at a final concentration of $2 \mathrm{mM}$.

Statistical Analysis. Statistical significance of the differences in APZ or ERY $\mathrm{CL}_{\text {int }}$ under varying concentrations of ERY were assessed by using a one-way ANOVA coupled with a Tukey's post hoc test. Statistical differences between mRNA content in control studies and those treated with escalating concentrations of ERY were assessed by using one-way ANOVA coupled with Dunnett's post hoc test. Statistical significance was defined as $P<0.05$.

Liquid Chromatography-Tandem Mass Spectrometry. Compound concentrations were measured by using liquid chromatography-tandem mass spectrometry on an Applied Biosystem Sciex API-4000 mass spectrometer coupled to a Waters Acquity ultra-high-performance liquid chromatography system or an Applied Biosystem Sciex API 6500 coupled to an Agilent Infinity 1290 HPLC in multiple reaction monitoring mode (MRM). Elution was carried out by using a linear gradient flow from $5 \%$ acetonitrile with $0.1 \%$ acetic acid in deionized water to $95 \%$ acetonitrile with $0.1 \%$ acetic acid in deionized water. For bioanalytical methods using the Waters Ultra Performance Liquid Chromatography system, separations were carried out with a Waters Acquity $\mathrm{BEH} \mathrm{C} \mathrm{C}_{18}$ column $(2.1 \times 50 \mathrm{~mm}, 1.7 \mu \mathrm{m})$, whereas bioanalyses using the Agilent HPLC system were carried out with an Agilent InfinityLab Poroshell $\mathrm{HPH}-\mathrm{C}_{18}, 1.9 \mu \mathrm{m}$, $(2.1 \times 50 \mathrm{~mm})$ column. Detection and quantification were carried out with MRM using known transitions shown in Table 2. In all methods for P450 substrates, the internal standard was nevirapine, measured using a mass-to-charge transition of $267 \rightarrow 226$ in positive mode. For UGT metabolites, the internal standard used was labetalol, measured using a mass-to-charge transition of $329.0 \rightarrow 294.3$ in positive mode.

\section{Results}

Inhibition of CYP3A Metabolism of APZ by KTZ. Coincubation of $1 \mu \mathrm{M} \mathrm{KTZ}$ with APZ in HepatoPac cultures (donor 1307) resulted in partial inhibition of APZ metabolism characterized by a biphasic curve with noticeable inhibition of APZ turnover up to 48 hours, followed by the resumption of turnover that was approximately equal to the control from 48 hours to the end of the incubation period (Fig. 1A). By 48 hours, KTZ levels were depleted to approximately $1 \%$ of nominal concentrations $(\sim 10 \mathrm{nM})$. Coincubation of $3 \mu \mathrm{M}$ of $\mathrm{KTZ}$ with APZ resulted in sustained inhibition of APZ metabolism throughout the incubation period. By 138 hours, KTZ levels were depleted to approximately $3 \%$ of nominal concentrations ( $90 \mathrm{nM})$ (Fig. 1B).

Inhibition of CYP3A Metabolism of APZ by ERY. Coincubation of ERY with APZ in HepatoPac cultures (donor 1307) resulted in an ERY concentration-dependent decrease in the $\mathrm{CL}_{\text {int }}$ of APZ (Fig. 2A). Unlike KTZ inhibition of APZ metabolism, the inhibition of APZ metabolism by ERY did not appear to be characterized by an initial slow rate of depletion followed by a later, more rapid rate of depletion. ERY turnover was concentration-dependent. At 3, 10, and $30 \mu \mathrm{M}$ ERY, 
TABLE 1

Hepatopac donor demographics

\begin{tabular}{lccc}
\hline Lot & Sex & Age & Cause of Death \\
\hline 1307 & Male & 31 & Anoxia, second to heroin overdose \\
3121 B & Female & 52 & Anoxia, second to cardiac arrest \\
4202 & Female & 39 & Anoxia, second to cardiac arrest \\
4297 & Female & 54 & Stroke \\
ACR (10-donor pool) & Mixed Sex & N/A \\
\hline
\end{tabular}

N/A, not applicable.

approximately $26 \%(0.78 \mu \mathrm{M}), 28 \%(2.8 \mu \mathrm{M})$, and $43 \%(22 \mu \mathrm{M})$ ERY remained, respectively, after 6 days.

Inhibition of CYP3A-Mediated Midazolam 1' Hydroxylation by ERY. ERY caused a dose- and time-dependent decrease in CYP3A activity in HepatoPac cultures (donor 1307, male, age 31, Caucasian) as measured by the formation of 1'OH midazolam (MDZ) from MDZ (Fig. 3A). Relatively stable inhibition of CYP3A activity was observed up to 72 hours for all concentrations of ERY. Inhibition of CYP3A activity was approximately $60 \%, 80 \%$, and $90 \%$ for 3,10 , and $30 \mu \mathrm{M}$ ERY, respectively. After 72 hours, however, CYP3A activity in the 3and $10-\mu \mathrm{M}$ treatment groups began recovering relative to the control, whereas the $30-\mu \mathrm{M}$ ERY treatment group maintained $>85 \%$ inhibition of CYP3A activity over the course of the incubation (Fig. 3B).

Evaluation of the Effect of ERY on Other P450 Isoforms. The effect of ERY on the $\mathrm{CL}_{\text {int }}$ of substrates of CYP1A2, CYP2C9, CYP2C19, and CYP2D6 was also evaluated at $30 \mu \mathrm{M}$ of ERY (Table 3). Mild inhibition was observed in the presence of $30 \mu \mathrm{M}$ of ERY for CYP2C9 (tolbutamide, 26\% inhibition) and CYP2D6 (risperidone, $21 \%$ inhibition) metabolism. Diazepam (2C19/3A) turnover was inhibited to a greater extent $(74 \%)$.

The inhibitory effect of ERY on APZ CL $\mathrm{L}_{\text {int }}$ was repeated with three to five additional donors at 15, 30, and $60 \mu \mathrm{M}$. At $30 \mu \mathrm{M}$ of ERY, APZ $\mathrm{CL}_{\text {int }}$ was, on average, inhibited by $>80 \%$ (Fig. $4 \mathrm{~A}$ ). The $\mathrm{CL}_{\text {int }}$ of ERY was approximately the same from 15 to $60 \mu \mathrm{M}$ of ERY (Fig. 4B).

Effect of ERY on Recombinant UGT Activity. The effect of ERY on the activity of recombinant UGTs 1A1, 1A3, 1A4, 1A6, 1A9, 2B7, and $2 \mathrm{~B} 15$ was evaluated at 30 and $60 \mu \mathrm{M}$ of ERY. Neither concentration of ERY was able to inhibit glucuronidation of B-estradiol glucuronidation by UGTs $1 \mathrm{~A} 1$ and $1 \mathrm{~A} 3$, trifluoperazine glucuronidation by UGT1A4, or 7-hydroxy-4-(trifluoromethyl) coumarin glucuronidation by UGTs 1A6, 1A9, 2B7, and 2B15 (data not shown).

Activation of Nuclear Receptors. ERY was assessed for its potential to activate the major nuclear receptors AhR, CAR, and PXR that are involved in the induction of drug metabolism enzymes in HepatoPac cultures (lot ACR). No statistically significant increase in CYP1A2 or 2B6 mRNA were observed at any concentration of ERY evaluated.
Statistically significant increases in CYP3A4 mRNA were observed at ERY concentrations of $60 \mu \mathrm{M}$ (1.8-fold) and $120 \mu \mathrm{M}$ (2.1-fold). In contrast, incubations with OME $(50 \mu \mathrm{M})$, CITCO $(0.1 \mu \mathrm{M})$, and RIF $(10 \mu \mathrm{M})$ were associated with markedly higher mRNA fold increases in CYP1A2 (102-fold), 2B6 (16-fold), and 3A4 (8.7-fold) (Fig. 5).

Effect of ERY on Hepatocyte Viability. Hepatocyte viability was assessed in the presence of 30 and $60 \mu \mathrm{M}$ of ERY by monitoring cell morphology and content of ALT in the cell fraction. Neither concentration of ERY was associated with a change in the morphology of the cells in comparison with the control (Fig. 6A). Furthermore, ERY was not shown to cause a change in ALT content in the cells. This is opposed to incubations with the positive control, acetaminophen $(20 \mathrm{mM})$, which resulted in a marked decrease in the concentration of hepatocyte ALT content (Fig. 6B).

\section{Discussion}

Identification of the metabolic enzymes responsible for the elimination of drug candidates, commonly referred to as reaction phenotyping, is an important step in determining victim DDI risk. Conventional in vitro systems to study reaction phenotyping include human liver microsomes (HLM) with specific inhibitors and recombinantly expressed enzymes. For moderate or high clearance compounds, compound depletion in HLM incubations in the presence of specific inhibitors of drug-metabolizing enzymes can provide the data necessary to assign $\mathrm{CL}_{\mathrm{int}}$ values to metabolic pathways in the liver. However, for more slowly cleared compounds, lack of sufficient metabolic turnover precludes any reliable calculation of changes in $\mathrm{CL}_{\mathrm{int}}$ in the presence of inhibitors. As such, determination of $\mathrm{CL}_{\text {int }}$ values for slowly cleared compounds would require synthesis of metabolite standards and extensive evaluation of metabolite formation kinetics, and resources to conduct such evaluations may not be available during early stage development.

The use of suspension hepatocytes for the conduct of CYP450 reaction phenotyping studies has been considered advantageous over the use of recombinant enzymes or microsomes, as hepatocytes in

TABLE 2

MRM transitions used for liquid chromatography-tandem mass spectrometry analysis

\begin{tabular}{lcc}
\hline Compound & MRM Transition & Enzyme \\
\hline Alprazolam & $309 \rightarrow 281(+)$ & CYP3A \\
Diazepam & $285 \rightarrow 193(+)$ & CYP2C19/CYP3A \\
Erythromycin & $735 \rightarrow 158(+)$ & CYP3A \\
Estradiol 3- $(\beta$-D-glucuronide) & $447.0 \rightarrow 271.1(-)$ & UGT1A1, UGT1A3 \\
Ketoconazole & $532 \rightarrow 489(+)$ & CYP3A \\
Midazolam & $342 \rightarrow 324(+)$ & CYP3A \\
Riluzole & $235 \rightarrow 166(+)$ & CYP1A2 \\
Risperidone & $411 \rightarrow 191(+)$ & CYP2D6 \\
Tolbutamide & $273 \rightarrow 155(+)$ & CYP2C9 \\
Trifluoromethylumbelliferyl $\beta$-D-glucuronide & $407 \rightarrow 231(+)$ & UGT1A6, UGT1A9, UGT2B7, UGT2B15 \\
Trifluoperazine N-glucuronide & $584.0 \rightarrow 408.1(+)$ & UGT1A4 \\
\hline
\end{tabular}



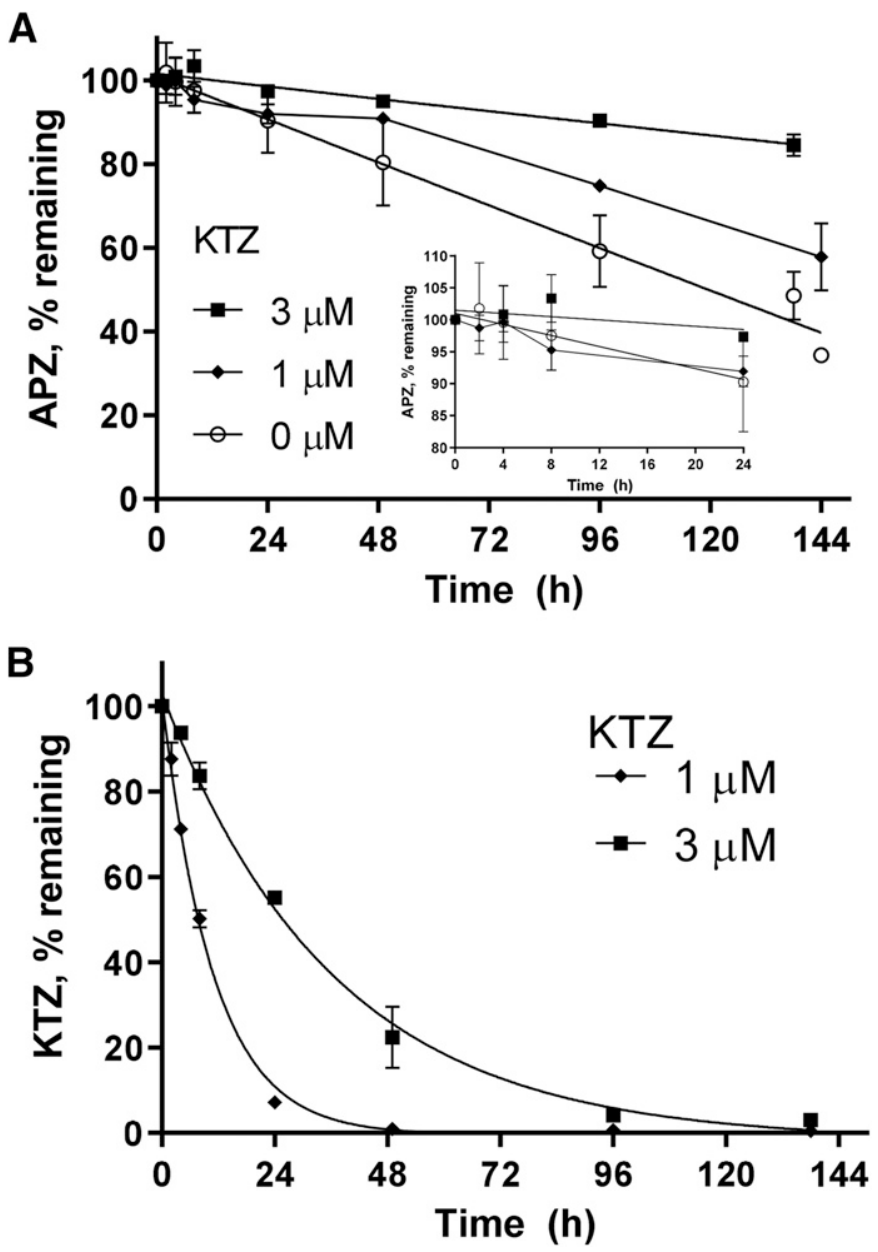

Fig. 1. Relationship between inhibition of APZ turnover (A) and KTZ exposure (B) in HepatoPac incubations (donor 1307). Values represent the mean \pm S.D. from three replicates.

suspension generally yield greater compound turnover (through extended incubation times) and possess the complete array of hepatic drug metabolizing enzymes (Argikar et al., 2016; Matsunaga et al., 2018). However, suspension hepatocytes are limited by the relatively short lifespan, i.e., they can only be used for a maximum of 4-6 hours (Gebhardt et al., 2003; Chan et al., 2013). Such limitations of incubation time with hepatocyte suspensions have been improved by periodically replenishing incubations with freshly prepared hepatocytes in the Hepatocyte Relay Method (Yang et al., 2016). Long-term hepatocyte cultures such as cocultures, flow systems, or spheroids permit lengthier incubation durations, providing novel ways to address metabolism questions for slowly cleared compounds. In this work, we sought to determine if the enhanced metabolic competency of a long-term hepatocyte model, HepatoPac, could provide additional utility as a model to investigate reaction phenotyping of slowly cleared drugs using a CYP3A-selective inhibitor.

Earlier use of the HepatoPac model in our laboratory involved incubation of the cells without FBS to mitigate drug binding to proteins during the incubation. Initial studies without FBS included incubations involving donors 1307, 3121B, and 4202. In later studies, it was determined that overall morphology of the cells, particularly for the supporting fibroblasts, could be improved by the inclusion of $5 \%$ serum (1.6-3.5 g/l albumin and $0.65 \mathrm{~g} / \mathrm{l})$, without adversely affecting metabolism of APZ $\left(f_{u, p}=0.29\right)$ and ERY $\left(f_{u, p}=0.36\right)$ (Prandota et al., 1980; Gstraunthaler, 2003). Nevertheless, the inclusion of
$5 \%$ serum can adversely affect the extent of depletion of substrates that are highly bound to plasma protein, which would decrease the ability to discern differences between treatments with and without ERY.

Ketoconazole. Even though potent inhibition of CYP3A in HepatoPac was observed in the presence of KTZ (Fig. 1A), it is clear that the relatively short-lived effect of $\mathrm{KTZ}(1 \mu \mathrm{M})$ on CYP3A is associated with its rapid elimination (Fig. 1B). Sustained inhibitory activity would likely require higher $\mathrm{KTZ}$ concentrations at the expense of inhibitor selectivity because ideal concentrations of KTZ for selective inhibition of CYP3A should not exceed $1 \mu \mathrm{M}$ in HLM (Bohnert et al., 2016). Alternatively, intermittent spiking of KTZ into the incubation could also achieve complete inhibition but was not evaluated in this study. For incubations with compounds that are more rapidly metabolized by hepatic CYP3A and that therefore do not require long incubation durations, KTZ can still provide a high degree of selective CYP3A inhibition. For more slowly eliminated compounds, the effect of the inhibitor must be sustained long enough for near-complete inhibition of the enzyme to discriminate parent turnover in the presence versus absence of the inhibitor. As such, the use of reversible inhibitors such as KTZ should not exceed durations that would lead to excessive depletion of KTZ and its associated inhibitory activity. In this study, the duration of effective CYP3A inhibition by $\mathrm{KTZ}$ at $1 \mu \mathrm{M}$ is 1 day. Assuming first-order elimination kinetics with a half-life of 8 hours, the $K_{\mathrm{i}}$ value $\mathrm{KTZ}(\sim 76 \mathrm{nM})$ (von Moltke et al., 1994) would be reached by approximately 31 hours after the start of the incubation. Using the same calculation method, if $3 \mu \mathrm{M}$ of KTZ is used, the $K_{\mathrm{i}}$ for KTZ should be reached by approximately 130 hours after the start of the incubation. These estimations are in good agreement with the data observed in this study (Fig. 1). At higher
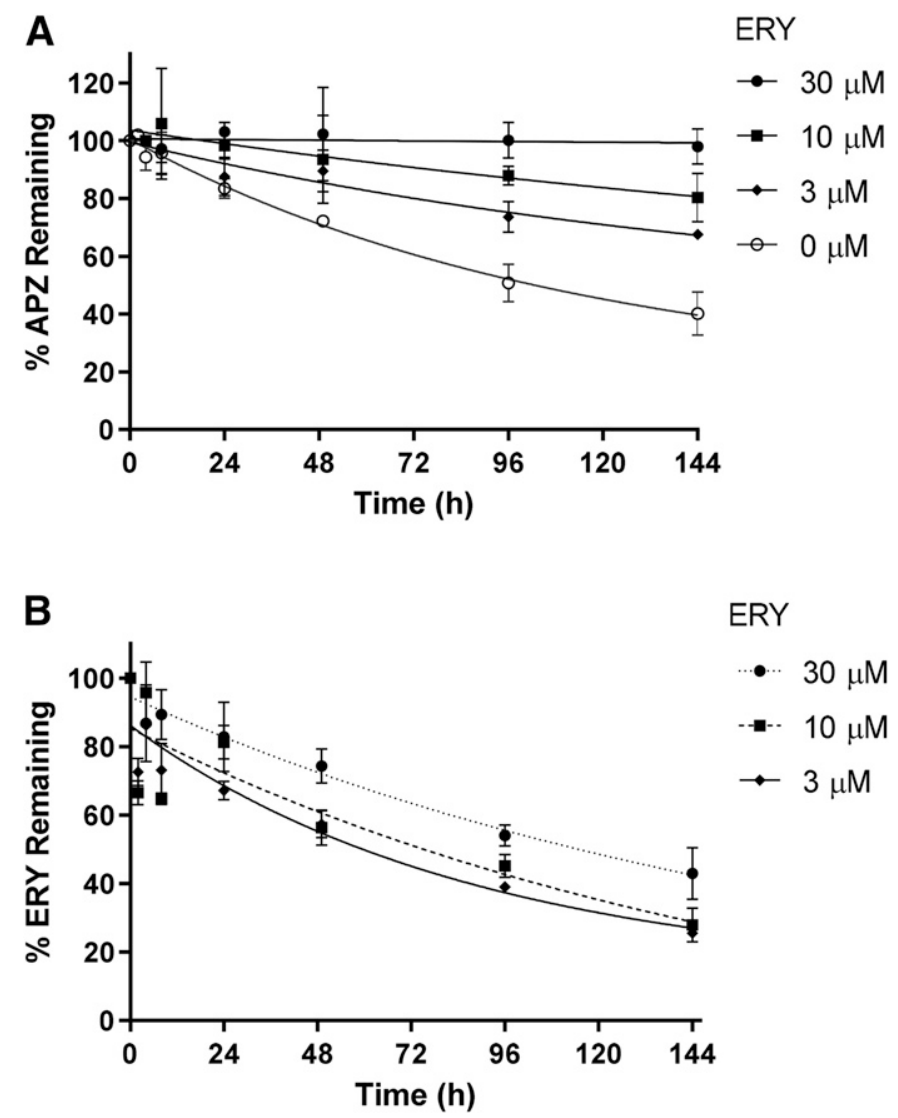

Fig. 2. Relationship between inhibition of APZ turnover (A) and ERY exposure (B) in HepatoPac incubations (donor 1307). Values represent the mean \pm S.D. from three replicates. 


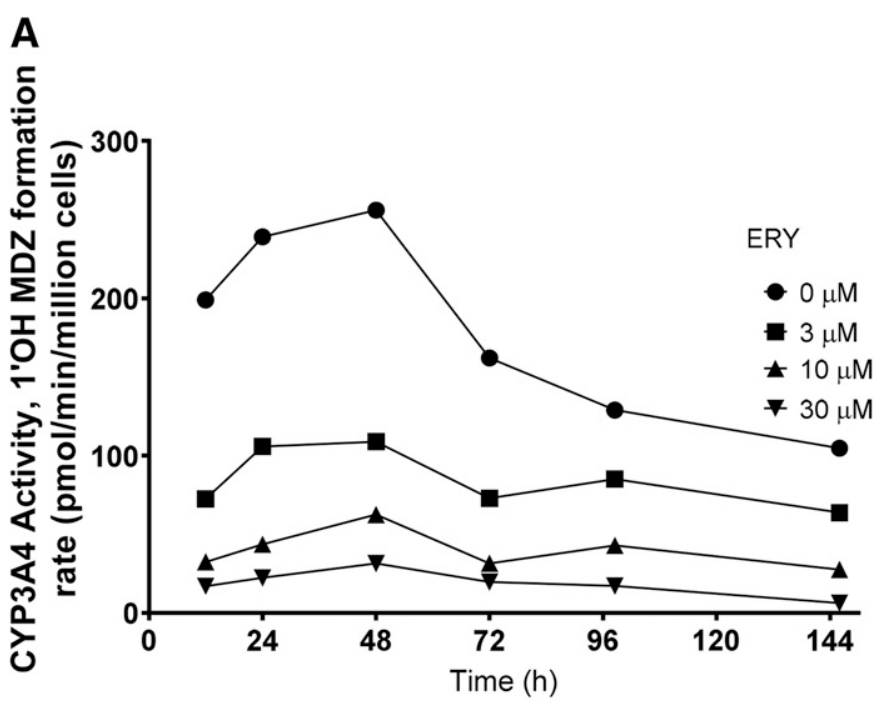

B

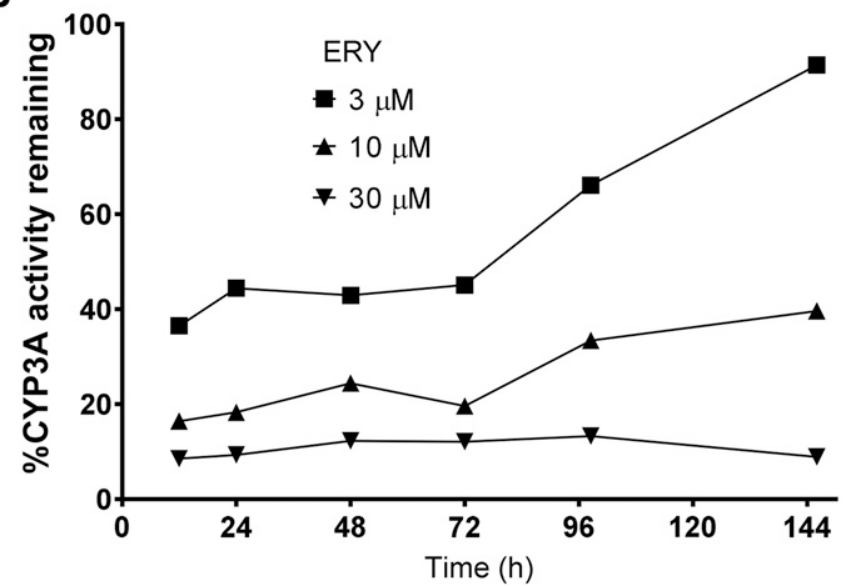

Fig. 3. Time course of CYP3A activity in the presence and absence of ERY. Values represent the mean of two replicates. (A) The time-dependent changes in $1^{\prime} \mathrm{OH}$ MDZ activity. (B) The percent remaining CYP3A activity in the presence of ERY relative to the control.

concentrations of KTZ, greater care should be exercised in accounting for loss of selectivity for CYP3A inhibition (Newton et al., 1995).

Erythromycin. ERY at concentrations tested up to $60 \mu \mathrm{M}$ was shown to be nontoxic to HepatoPac cultures over an incubation duration of up to 144 hours (Fig. 6), confirming the absence of any confounding effects it may have on viability of the cells. Time-dependent inactivation of CYP3A, which requires enzyme resynthesis as well as inactivator depletion to recover CYP3A activity, should result in a longer lasting inhibition of CYP3A activity compared with reversible inhibitors (Di, 2017). The $K_{\mathrm{I}}$ and $k_{\text {inact }}$ values for ERY in HLM are $5.3 \mu \mathrm{M}$ and 0.06 minutes $^{-1}$, respectively (Chen et al., 2011). First-order loss of enzyme activity through inactivation can be described by the MichaelisMenten model, where $\lambda$ is the rate constant for loss of CYP3A activity and assuming complete permeability of ERY and full access to the CYP3A active site (eq. 1):

$$
\lambda=\frac{[E R Y] \times k_{\text {inact }}}{[E R Y]+K_{I}} .
$$

At $20 \mu \mathrm{M}$ of ERY (average concentration in incubation with $30 \mu \mathrm{M}$ of ERY for donor 1307), CYP3A activity should be diminished to approximately 1\%, 3.5 hours after the start of the incubation (Mayhew et al., 2000). This is a relatively short period of time in comparison with the full incubation period of 6 to 7 days. Even at $0.78 \mu \mathrm{M}$ of ERY, which is the concentration of ERY remaining at the end of the incubation with $3 \mu \mathrm{M}$ of ERY, CYP3A activity should have been diminished to approximately $1 \%$ by 10 hours, using the HLM parameters shown above. In the same study, inactivation parameters generated by using cryopreserved human hepatocytes in suspension were found to have approximately 10 -fold lower inactivation efficiency, which was mainly due to a higher $K_{\mathrm{I}}$ value (Chen et al., 2011). Although not evaluated, the authors hypothesized that the discrepancy between observed inactivation in microsomes and hepatocytes could be due to nonspecific binding, transport, or metabolism that is not present in the microsomal system. Using ERY inactivation parameters from these hepatocyte studies, a $25 \%$ loss of CYP3A4 activity would be expected by 48 hours using $3 \mu \mathrm{M}$ of ERY, which is more consistent with the observations in this study. The discrepancy between the lower levels of inhibition observed in this study and the estimated calculations from parameters derived from HLM experiments could also be due to enzyme synthesis (Chan et al., 2018), which has also been shown to occur in the HepatoPac model (Ramsden et al., 2015). In addition, inhibitors that can activate nuclear receptors (e.g., ritonavir) can increase the synthesis rate of enzymes, further complicating the interpretation of the effect of the inhibitor on induced enzymes. In this study, ERY has been shown to cause very mild in vitro induction of CYP3A4 at concentrations up to $120 \mu \mathrm{M}$ (Fig. 5C). It is possible that this mild level of induction could also counteract the inactivation of CYP3A, thus adding to the discrepancy between expected inhibition of CYP3A based on HLM data. ERY was not shown to activate AhR and CAR in this study as defined by mRNA fold increases in CYP1A2 and 2B6 that were $\geq 2$. Furthermore, for CYP2B6, increases in mRNA content at any concentration of ERY evaluated were not found to be significantly different than the vehicle control. PXR activation as defined by a 2-fold increase in CYP3A4 mRNA was also not observed at up to $60 \mu \mathrm{M}$ of ERY,

TABLE 3

Effect of ERY on other CYP450 isoforms

For incubations with diazepam, riluzole, and risperidone, $\mathrm{CL}_{\text {int }}$ values were derived from the average of two replicates using donor 1307. Tolbutamide $\mathrm{CL}_{\mathrm{int}}$ values were derived from the average of three replicates each for donors 1307 and ACR. APZ CL $\mathrm{L}_{\text {int }}$ values were derived from the average of two to five replicates each from donors 1307, 3121B, 4202, 4297, and ACR.

\begin{tabular}{lcccc}
$\begin{array}{l}\text { Probe } \\
\text { Substrate }\end{array}$ & $\begin{array}{c}\text { P450 Responsible for } \\
\text { Metabolism }\end{array}$ & $\begin{array}{c}\text { Percent Metabolized by } \\
\text { Isoform }\end{array}$ & $\begin{array}{c}\text { Expected Percent Inhibition } \\
\text { by } 30 \mu \mathrm{M} \text { ERY }\end{array}$ & $\begin{array}{c}\text { Observed Percent Inhibition } \\
\text { by 30 } \mu \mathrm{M} \text { ERY }\end{array}$ \\
\hline Riluzole & CYP2D6 & $>80$ & 0 & 7.7 \\
Tolbutamide & CYP2C9 & $>85$ & 0 & 26 \\
Diazepam & CYP3A4/CYP2C19 & $60 / 40$ & 60 & 74 \\
Risperidone & CYP2D6 & $>93$ & 100 & 21 \\
Alprazolam & CYP3A4 & $>90$ & 11 \\
\hline
\end{tabular}


A
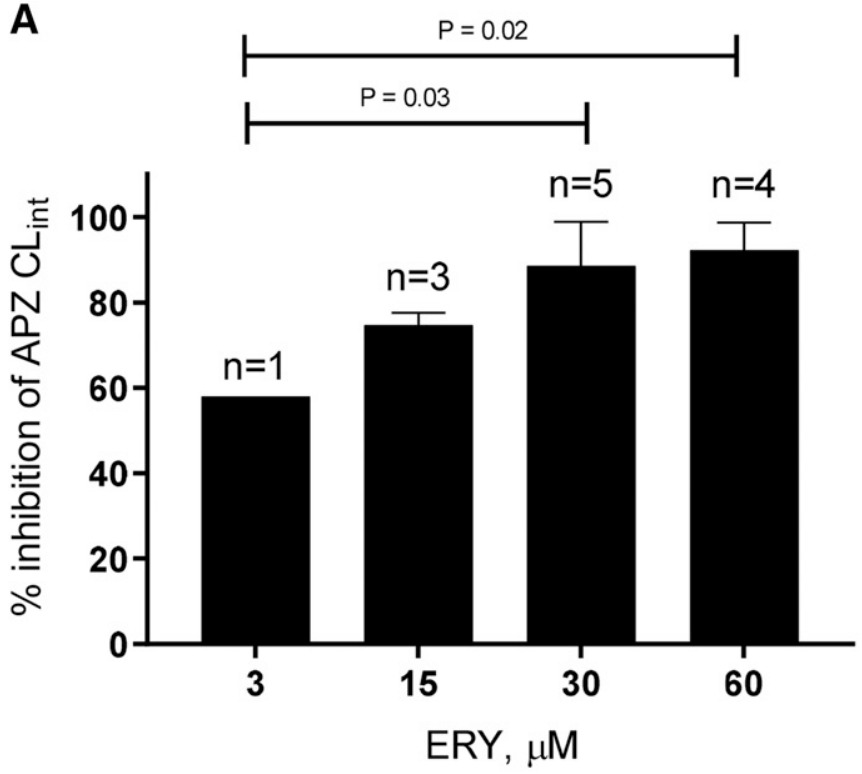

B

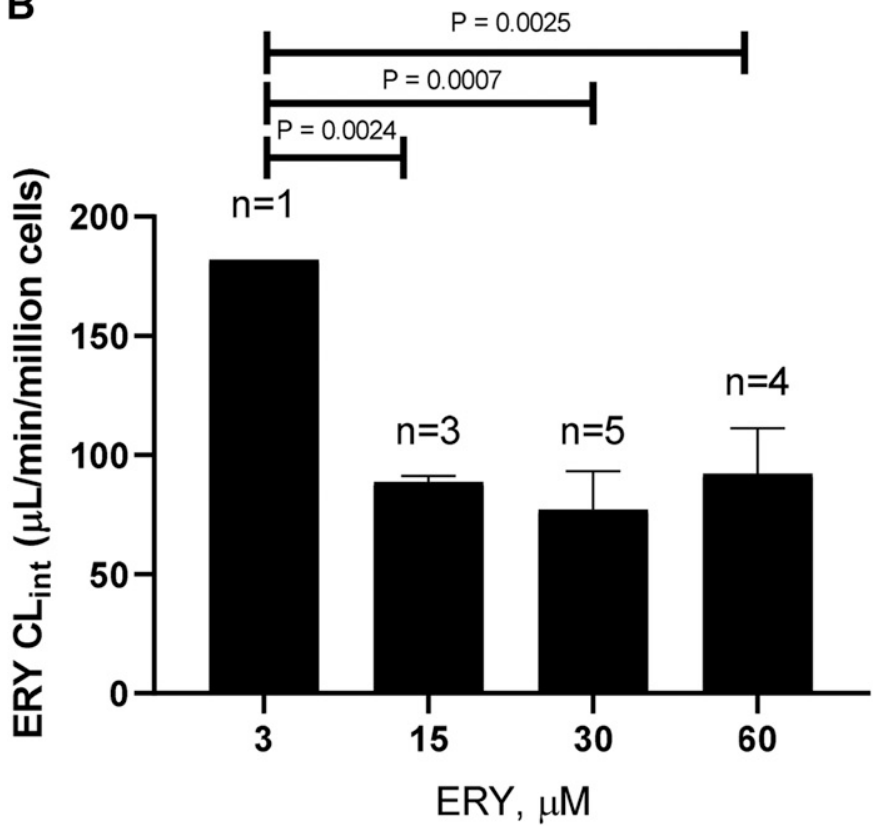

Fig. 4. (A) Percent inhibition of $A P Z C_{\text {int }}$ by ERY in HepatoPac cultures from multiple donors. (B) ERY $\mathrm{CL}_{\text {int }}$ from multiple donors at various concentrations of ERY. Values represent the mean \pm S.D. of the number of donors used.

whereas $120 \mu \mathrm{M}$ of ERY only resulted in a 2.1-fold increase in CYP3A4 mRNA (Fig. 5). These results are consistent with a previous study demonstrating that the inducing effects of ERY on drug metabolizing enzymes appear to be markedly lower in comparison with other macrolide antibiotic CYP3A inactivators, such as troleandomycin (Ledirac et al., 2000). Sustained inhibition of CYP3A activity was observed with ERY concentrations $\geq 30 \mu \mathrm{M}$ (Figs. $2 \mathrm{~A}, 3 \mathrm{~B}$, and $4 \mathrm{~A}$ ) with minimal increase in CYP3A gene expression (1.8-fold) or significant loss in hepatocyte viability (Fig. 6), suggesting that this is an ideal concentration of ERY to be used for selectively inhibiting CYP3A in vitro. It is important to emphasize that these interpretations are confined to the use of ERY as an in vitro tool in long-term cultures such as HepatoPac and should not be interpreted in the context of its use as clinical DDI tool.
ERY is also a relatively selective inhibitor of CYP3A in the HepatoPac model as indicated by weak to no inhibition of $\mathrm{CL}_{\text {int }}$ for substrates of several other major CYP450s (Table 2). Marked inhibition of diazepam $\mathrm{CL}_{\mathrm{int}}$ could be explained by data showing that diazepam is metabolized partly by CYP3A (60\%) and CYP2C19 (40\%) (Andersson et al., 1994). It is more difficult to understand why ERY inhibited tolbutamide clearance by $26 \%$ and riluzole clearance by $21 \%$; to our

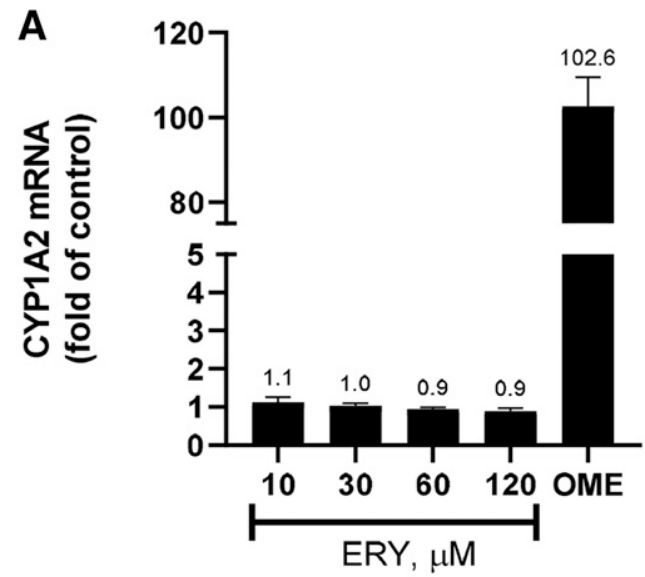

B
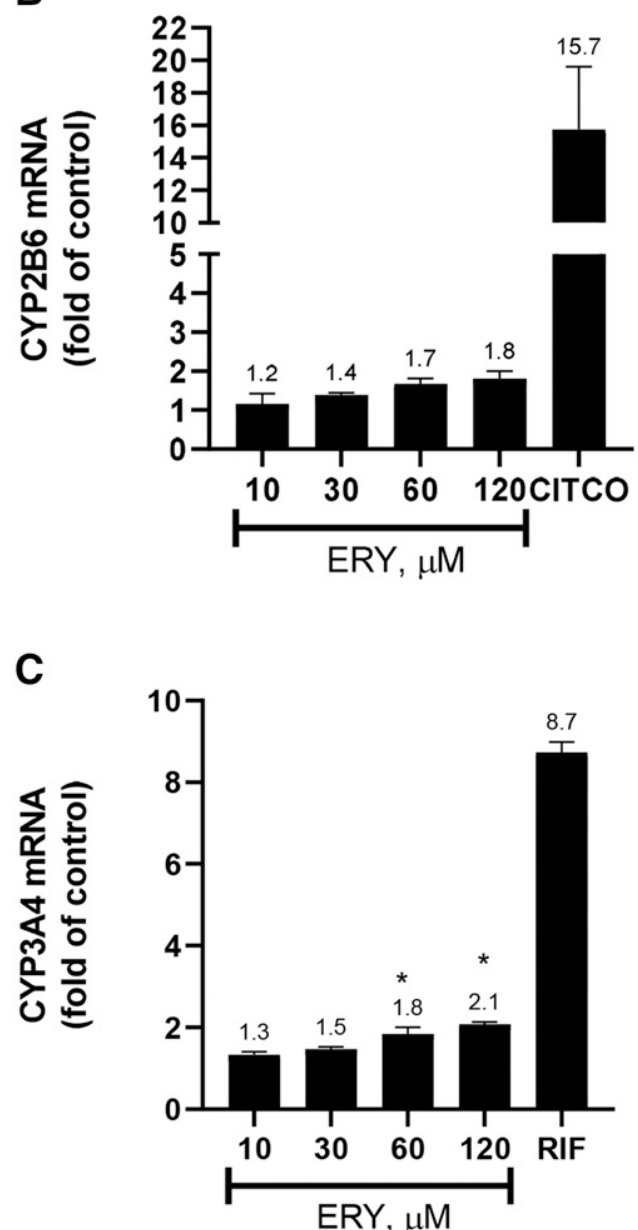

Fig. 5. CYP1A2 (A), CYP2B6 (B), and CYP3A4 (C) expression in the presence of up to $120 \mu \mathrm{M}$ ERY, $50 \mu \mathrm{M}$ OME, and $10 \mu \mathrm{M}$ RIF. Values are depicted as the mean \pm S.D. of three individual biologic replicates. $* P<0.05$, statistically significant induction relative to the vehicle control. 
A

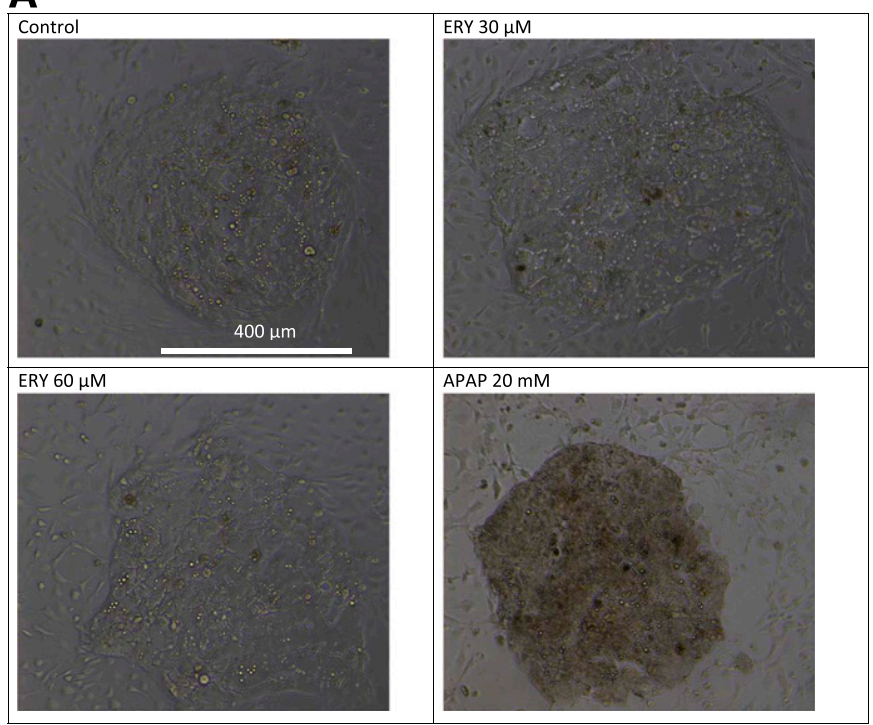

B

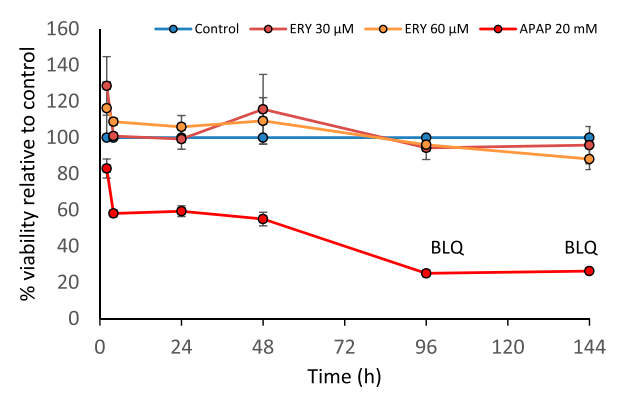

Fig. 6. (A) Comparison of hepatocyte island morphology in the vehicle-treated control vs. hepatocyte island morphology in the presence of ERY $(30$ or $60 \mu \mathrm{M})$ and APAP $(20 \mathrm{mM}) 144$ hours after start of the incubation. (B) Hepatocellular ALT content over the course of a 144 hours incubation (donor ACR). APAP, acetaminophen.

knowledge, neither tolbutamide or riluzole have been reported to be substrates of CYP3A, and ERY has not been shown to be an inhibitor of CYP1A or CYP2C isoforms. Early studies in HLM have demonstrated inhibitory potential of KTZ on tolbutamide metabolism but with a very high $\mathrm{IC}_{50}$ value of $18 \mu \mathrm{M}$ (Back et al., 1989). Also, the metabolism of the anticancer agent, 5,6-dimethyxanthenone-4-acetic acid (DMXAA), a substrate of CYP1A2, was shown to be inhibited by ERY by up to $31 \%$ in HLM, but only at a very high concentration $(500 \mu \mathrm{M})$. Essentially no inhibition of DMXAA metabolism was observed at $100 \mu \mathrm{M}$ ERY. Further assessment of the potential inhibition of CYP1A and CYP2C9 by ERY in HepatoPac cultures could benefit from the use of other CYP2C9 substrates and HepatoPac donors, particularly because the ERY has already been used in in vitro studies to help determine CYP3A contribution to metabolism. Some examples of the in vitro use of ERY as a selective CYP3A inhibitor include the anticancer drug 5,6DMXAA, the analgesic, elitriptan, and dihydrocodeine (Kirkwood et al., 1997; Zhou et al., 2000; Evans et al., 2003). Regulatory authorities also list ERY as a prototypical moderate CYP3A inhibitor for use in clinical studies. Because this is the first time that ERY has been used as an inhibitor in the HepatoPac model, it is possible that distinguishing characteristics of the model could lead to CYP1A and 2C results, which are not completely consistent with more conventional models.

With respect to non-P450 drug-metabolizing enzymes, results using recombinant UGTs in this study demonstrated that ERY is also not likely to inhibit major UGT isoforms. Consistent with this, Takeda et al. (2006) reported that ERY did not inhibit UGT2B7 morphine glucuronidation. ERY however, has been shown to possess inhibitory activity against aldehyde oxidase ( $\mathrm{IC}_{50}=15 \mu \mathrm{M}$ in HLM) (Obach et al., 2004) and P-glycoprotein (P-gp) (Eriksson et al., 2006; Eberl et al., 2007). As such, caution should be exercised in interpreting results using ERY when evaluated with a substrate of aldehyde oxidase or P-gp. P-gp inhibition could potentially alter metabolic turnover of compounds through alteration of intracellular concentrations.

In summary, based on its sustained inhibition of CYP3A, mild ability to activate PXR, relative selectivity, and tolerability in cell cultures, ERY may be a useful tool in combination with long-term hepatocyte cultures to study the contribution of CYP3A to overall hepatic metabolism of low-clearance compounds. The use of metabolically active, long-term models such as HepatoPac for reaction phenotyping studies can further benefit from the discovery of inhibitors with greater potency, selectivity. and stability (Khojasteh et al., 2011; Di, 2017).

\section{Authorship Contributions}

Participated in research design: Chan.

Conducted experiments: Chan, Scaringella, Raymond.

Performed data analysis: Chan, Scaringella, Raymond.

Wrote or contributed to the writing of the manuscript: Chan, Taub.

\section{References}

Andersson T, Miners JO, Veronese ME, and Birkett DJ (1994) Diazepam metabolism by human liver microsomes is mediated by both S-mephenytoin hydroxylase and CYP3A isoforms. $\mathrm{Br}$ J Clin Pharmacol 38:131-137.

Argikar UA, Potter PM, Hutzler JM, and Marathe PH (2016) Challenges and opportunities with non-CYP enzymes aldehyde oxidase, carboxylesterase, and UDP-glucuronosyltransferase: focus on reaction phenotyping and prediction of human clearance. AAPS J 18:1391-1405.

Back DJ, Stevenson P, and Tjia JF (1989) Comparative effects of two antimycotic agents, ketoconazole and terbinafine on the metabolism of tolbutamide, ethinyloestradiol, cyclosporin and ethoxycoumarin by human liver microsomes in vitro. Br J Clin Pharmacol 28:166-170.

Beckwitt CH, Clark AM, Wheeler S, Taylor DL, Stolz DB, Griffith L, and Wells A (2018) Liver 'organ on a chip'. Exp Cell Res 363:15-25.

Bohnert T, Patel A, Templeton I, Chen Y, Lu C, Lai G, Leung L, Tse S, Einolf HJ, Wang Y-H, et al.; International Consortium for Innovation and Quality in Pharmaceutical Development (IQ) Victim Drug-Drug Interactions Working Group (2016) Evaluation of a new molecular entity as a victim of metabolic drug-drug interactions-an industry perspective. Drug Metab Dispos 44: 1399-1423.

Cerny MA (2016) Prevalence of non-cytochrome P450-mediated metabolism in food and drug administration-approved oral and intravenous drugs: 2006-2015. Drug Metab Dispos 44: 1246-1252.

Chan CYS, Roberts O, Rajoli RKR, Liptrott NJ, Siccardi M, Almond L, and Owen A (2018) Derivation of CYP3A4 and CYP2B6 degradation rate constants in primary human hepatocytes: a siRNA-silencing-based approach. Drug Metab Pharmacokinet 33:179-187.

Chan TS, Yu H, Moore A, Khetani SR, and Tweedie D (2013) Meeting the challenge of predicting hepatic clearance of compounds slowly metabolized by cytochrome P450 using a novel hepatocyte model, HepatoPac [published correction appears in Drug Metab Dispos (2014) 42:200; Drug Metab Dispos (2019) 47:58-66]. Drug Metab Dispos 41:2024-2032.

Chen Y, Liu L, Monshouwer M, and Fretland AJ (2011) Determination of time-dependent inactivation of CYP3A4 in cryopreserved human hepatocytes and assessment of human drug-drug interactions. Drug Metab Dispos 39:2085-2092.

Di L (2017) Reaction phenotyping to assess victim drug-drug interaction risks. Expert Opin Drug Discov 12:1105-1115.

Eberl S, Renner B, Neubert A, Reisig M, Bachmakov I, König J, Dörje F, Mürdter TE, Ackermann A, Dormann H, et al. (2007) Role of p-glycoprotein inhibition for drug interactions: evidence from in vitro and pharmacoepidemiological studies. Clin Pharmacokinet 46:1039-1049.

Eriksson UG, Dorani H, Karlsson J, Fritsch H, Hoffmann KJ, Olsson L, Sarich TC, Wall U, and Schützer KM (2006) Influence of erythromycin on the pharmacokinetics of ximelagatran may involve inhibition of P-glycoprotein-mediated excretion. Drug Metab Dispos 34:775-782.

Evans DC, O'Connor D, Lake BG, Evers R, Allen C, and Hargreaves R (2003) Eletriptan metabolism by human hepatic CYP450 enzymes and transport by human P-glycoprotein. Drug Metab Dispos 31:861-869.

Gebhardt R, Hengstler JG, Müller D, Glöckner R, Buenning P, Laube B, Schmelzer E, Ullrich M, Utesch D, Hewitt N, et al. (2003) New hepatocyte in vitro systems for drug metabolism: metabolic capacity and recommendations for application in basic research and drug development, standard operation procedures. Drug Metab Rev 35:145-213.

Gstraunthaler G (2003) Alternatives to the use of fetal bovine serum: serum-free cell culture. ALTEX 20:275-281.

Guengerich FP (1999) Cytochrome P-450 3A4: regulation and role in drug metabolism. Annu Rev Pharmacol Toxicol 39:1-17.

Jones BC, Middleton DS, and Youdim K (2009) Cytochrome P450 metabolism and inhibition: analysis for drug discovery. Prog Med Chem 47:239-263.

Khetani SR and Bhatia SN (2008) Microscale culture of human liver cells for drug development. Nat Biotechnol 26:120-126.

Khojasteh SC, Prabhu S, Kenny JR, Halladay JS, and Lu AY (2011) Chemical inhibitors of cytochrome P450 isoforms in human liver microsomes: a re-evaluation of P450 isoform selectivity. Eur J Drug Metab Pharmacokinet 36:1-16. 
Kirkwood LC, Nation RL, and Somogyi AA (1997) Characterization of the human cytochrome P450 enzymes involved in the metabolism of dihydrocodeine. Br J Clin Pharmacol 44:549-555.

Ledirac N, de Sousa G, Fontaine F, Agouridas C, Gugenheim J, Lorenzon G, and Rahmani R (2000) Effects of macrolide antibiotics on CYP3A expression in human and rat hepatocytes: interspecies differences in response to troleandomycin. Drug Metab Dispos 28:1391-1393.

Lin C, Shi J, Moore A, and Khetani SR (2016) Prediction of drug clearance and drug-drug interactions in microscale cultures of human hepatocytes. Drug Metab Dispos 44:127-136.

Livak KJ and Schmittgen TD (2001) Analysis of relative gene expression data using real-time quantitative PCR and the 2(-Delta Delta C(T)) method. Methods 25:402-408.

Matsunaga N, Fukuchi Y, Imawaka H, and Tamai I (2018) Sandwich-cultured hepatocytes for mechanistic understanding of hepatic disposition of parent drugs and metabolites by transporterenzyme interplay. Drug Metab Dispos 46:680-691.

Mayhew BS, Jones DR, and Hall SD (2000) An in vitro model for predicting in vivo inhibition of cytochrome P450 3A4 by metabolic intermediate complex formation. Drug Metab Dispos 28 1031-1037.

Newton DJ, Wang RW, and Lu AY (1995) Cytochrome P450 inhibitors. Evaluation of specificities in the in vitrometabolism of therapeutic agents by human liver microsomes. Drug Metab Dispos 23:154-158.

Obach RS, Huynh P, Allen MC, and Beedham C (2004) Human liver aldehyde oxidase: inhibition by 239 drugs. J Clin Pharmacol 44:7-19.

Prandota J, Tillement JP, d'Athis P, Campos H, and Barre J (1980) Binding of erythromycin base to human plasma proteins. J Int Med Res 8 (Suppl 2):1-8.

Ramsden D, Zhou J, and Tweedie DJ (2015) Determination of a degradation constant for CYP3A4 by direct suppression of mRNA in a novel human hepatocyte model, HepatoPac. Drug Metab Dispos 43:1307-1315.

Sohlenius-Sternbeck AK, Afzelius L, Prusis P, Neelissen J, Hoogstraate J, Johansson J, Floby E, Bengtsson A, Gissberg O, Sternbeck J, et al. (2010) Evaluation of the human prediction of clearance from hepatocyte and microsome intrinsic clearance for 52 drug compounds. Xenobiotica 40:637-649.

Takeda S, Kitajima Y, Ishii Y, Nishimura Y, Mackenzie PI, Oguri K, and Yamada H (2006) Inhibition of UDP-glucuronosyltransferase $2 \mathrm{~b} 7$-catalyzed morphine glucuronidation by ketoconazole: dual mechanisms involving a novel noncompetitive mode. Drug Metab Dispos 34 $1277-1282$.

von Moltke LL, Greenblatt DJ, Cotreau-Bibbo MM, Harmatz JS, and Shader RI (1994) Inhibitors of alprazolam metabolism in vitro: effect of serotonin-reuptake-inhibitor antidepressants, ketoconazole and quinidine. Br J Clin Pharmacol 38:23-31.

Yan Z and Caldwell GW (2013) In vitro identification of cytochrome P450 enzymes responsible for drug metabolism. Methods Mol Biol 1015:251-261.

Yang X, Atkinson K, and Di L (2016) Novel cytochrome P450 reaction phenotyping for lowclearance compounds using the hepatocyte relay method. Drug Metab Dispos 44:460-465.

Zhang H, Davis CD, Sinz MW, and Rodrigues AD (2007) Cytochrome P450 reaction-phenotyping: an industrial perspective. Expert Opin Drug Metab Toxicol 3:667-687.

Zhou S, Paxton JW, Tingle MD, and Kestell P (2000) Identification of the human liver cytochrome P450 isoenzyme responsible for the 6-methylhydroxylation of the novel anticancer drug 5,6dimethylxanthenone-4-acetic acid. Drug Metab Dispos 28:1449-1456.

Zientek MA and Youdim K (2015) Reaction phenotyping: advances in the experimental strategies used to characterize the contribution of drug-metabolizing enzymes. Drug Metab Dispos 43:163-181.

Address correspondence to: Tom S. Chan, Department of Drug Metabolism and Pharmacokinetics, Boehringer Ingelheim Pharmaceuticals Inc., 900 Ridgebury Rd., Ridgefield, CT 06877. E-mail: tom.chan@boehringer-ingelheim.com 